\title{
CONTRIBUTION TO THE JACOBIAN CONJECTURE: POLYNOMIAL MAPPING HAVING TWO ZEROS AT INFINITY
}

\author{
Grzegorz Biernat, Sylwia Lara-Dziembek, Edyta Pawlak \\ Institute of Mathematics, Czestochowa University of Technology, \\ Czestochowa, Poland \\ grzegorz.biernat@im.pcz.pl,sylwia.lara@im.pcz.pl,edyta.pawlak@im.pcz.pl
}

\begin{abstract}
This article contains the theorems concerning the algebraic dependence of polynomial mappings with the constant Jacobian having two zeros at infinity. The work is related to the issues of the classical Jacobian Conjecture. This hypothesis affirm that the polynomial mapping of two complex variables with constant non-zero Jacobian is invertible. The Jacobian Conjecture is equivalent to the fact that polynomial mappings with constant non-zero Jacobian do not have two zeros at infinity, therefore it is equivalent to the two theorems given in the work. The proofs of these theorems proceeds by induction.
\end{abstract}

Keywords: zeros at infinity, Jacobian Conjecture

\section{Introduction}

The hypothesis formulated by O.H.Keller in 1939 [1] that a polynomial mapping of constant Jacobian is invertible is not up to now resolved. Even in the case of two variables. This classic case is the subject of this work. In 1974, T.T.Moh in work [2] formulated a known condition for the invertibility of the mapping that additionally having one branch at infinity. A certain contribution in this topic was given by the co-author of this article at work [3]. Finally, in 1977 S.S. Abhyankar at work [4] proved that the polynomial mapping with constant Jacobian has at most two zeros at infinity. This work is particularly important for the authors of this article, because the theorems we prove excludes this possibility. It is worth mentioning about the work [5], of which Z.Charzyński was one of the co-authors. In the aforementioned work [4], Abhyankar showed that the mapping of the constant Jacobian having one zero at infinity is inveertible. The Jacobian Conjecture was also the subject of articles by D. Wright [6] and A. van den Essen [7]. The article [8] by H. Bass, E. Connell and D. Wright is also highlighted.

\section{Algebraic dependence of polynomial mappings}

Let $f_{i}, h_{j}$ be the complex forms of variables $X, Y$ of degrees $i, j$ respectively and $i, j \geq 1$.

Theorem I. Let

$$
f=(X Y)^{p}+f_{2 p-1}+f_{2 p-2}+f_{2 p-3}+\ldots+f_{1}
$$

and

$$
h=(X Y)^{q}+h_{2 q-1}+h_{2 q-2}+h_{2 q-3}+\ldots+h_{1}
$$

where $p \geq q \geq 1$. 
If $\operatorname{Jac}(f, h)=$ const $=\operatorname{Jac}\left(f_{1}, h_{1}\right)$ then exist the form $\hat{h}_{1}$ for which

$$
f=\left(X Y+\frac{1}{q} \hat{h}_{1}\right)^{p}+A_{1}\left(X Y+\frac{1}{q} \hat{h}_{1}\right)^{p-1}+\ldots+A_{p-1}\left(X Y+\frac{1}{q} \hat{h}_{1}\right)
$$

and

$$
h=\left(X Y+\frac{1}{q} \hat{h}_{1}\right)^{q}+B_{1}\left(X Y+\frac{1}{q} \hat{h}_{1}\right)^{q-1}+\ldots+B_{q-1}\left(X Y+\frac{1}{q} \hat{h}_{1}\right)
$$

for some constants $A_{1}, \ldots, A_{p-1}$ and $B_{1}, \ldots, B_{q-1}$.

Proof. For $q=1$ the theorem is true. Indeed, when

$$
f=(X Y)^{p}+f_{2 p-1}+f_{2 p-2}+f_{2 p-3}+\ldots+f_{1}
$$

and

$$
h=X Y+h_{1}
$$

so $\hat{h}_{1}=h_{1}$.

For $p=1$ the theorem is true, because then $f=h$.

Let $p \geq 2$. We assume that the formula (3) is true for exponents $1, \ldots, p-1$, and we prove that it is true for $p$.

Really, let

$$
f=(X Y)^{p}+\left.f_{2 p-1}\right|^{1)}+\left.f_{2 p-2}\right|^{2)}+\left.f_{2 p-3}\right|^{3)}+\ldots+\left.f_{1}\right|^{2 p-1)}
$$

and

$$
h=X Y+\left.h_{1}\right|^{1)}+\left.0\right|^{2)}+\left.0\right|^{3)}+\ldots+\left.0\right|^{2 p-1)}
$$

We have

$$
\text { 1) } \operatorname{Jac}\left((X Y)^{p}, h_{1}\right)=\operatorname{Jac}\left(X Y, f_{2 p-1}\right)
$$

so

$$
p(X Y)^{p-1} \operatorname{Jac}\left(X Y, h_{1}\right)=\operatorname{Jac}\left(X Y, f_{2 p-1}\right)
$$

therefore

$$
p(X Y)^{p-1} h_{1}=f_{2 p-1}
$$

Next

$$
\text { 2) } \underbrace{\operatorname{Jac}\left(f_{2 p-1}, h_{1}\right)}_{1^{\circ}}=\operatorname{Jac}\left(X Y, f_{2 p-2}\right)
$$


where

$$
1^{\circ}=\operatorname{Jac}\left(f_{2 p-1}, h_{1}\right)=\operatorname{Jac}\left(p(X Y)^{p-1} h_{1}, h_{1}\right)=p(p-1)(X Y)^{p-2} h_{1} \operatorname{Jac}\left(X Y, h_{1}\right)
$$

and back to the previous formula, we have

$$
p(p-1)(X Y)^{p-2} h_{1} \mathrm{Jac}\left(X Y, h_{1}\right)=\operatorname{Jac}\left(X Y, f_{2 p-2}\right)
$$

so

$$
\left(\begin{array}{l}
p \\
2
\end{array}\right)(X Y)^{p-2} h_{1}^{2}+A_{1}(X Y)^{p-1}=f_{2 p-2}
$$

Back to the coordinates $f$ and $h$, consecutively we obtain

$$
\begin{aligned}
& f=(X Y)^{p}+p(X Y)^{p-1} h_{1}+\frac{p(p-1)}{2}(X Y)^{p-2} h_{1}^{2}+A_{1}(X Y)^{p-1}+f_{2 p-3}+\ldots+f_{p}+\ldots+f_{1} \\
& h^{p}=\left(X Y+h_{1}\right)^{p}=(X Y)^{p}+\left(\begin{array}{c}
p \\
1
\end{array}\right)(X Y)^{p-1} h_{1}+\left(\begin{array}{l}
p \\
2
\end{array}\right)(X Y)^{p-2} h_{1}^{2}+\left(\begin{array}{l}
p \\
3
\end{array}\right)(X Y)^{p-3} h_{1}^{3}+\ldots+h_{1}^{p}
\end{aligned}
$$

so subtracting the above equations we get

$$
f-h^{p}=A_{1}(X Y)^{p-1}+\left(f_{2 p-3}-\left(\begin{array}{l}
p \\
3
\end{array}\right)(X Y)^{p-3} h_{1}^{3}\right)+\ldots+\left(f_{p}-h_{1}^{p}\right)+f_{p-1}+\ldots+f_{1}
$$

Let $A_{1} \neq 0$. Denote

$$
\begin{aligned}
& \tilde{f}=\frac{1}{A_{1}}\left(f-h^{p}\right)=(X Y)^{p-1}+\frac{1}{A_{1}}\left(\left(f_{2 p-3}-\left(\begin{array}{l}
p \\
3
\end{array}\right)(X Y)^{p-3} h_{1}^{3}\right)+\ldots+\left(f_{p}-h_{1}^{p}\right)+f_{p-1}+\ldots+f_{1}\right)= \\
& =(X Y)^{p-1}+\frac{1}{A_{1}}\left(\tilde{f}_{2 p-3}+\ldots+\tilde{f}_{p}+f_{p-1}+\ldots+f_{1}\right)
\end{aligned}
$$

Using the induction assumption for exponent $p-1$ we have

$$
\tilde{f}=h^{p-1}+\tilde{A}_{1} h^{p-2}+\ldots+\tilde{A}_{p-2} h
$$

so

$$
\begin{gathered}
f=h^{p}+A_{1} \tilde{f} \\
f=h^{p}+A_{1}\left(h^{p-1}+\tilde{A}_{1} h^{p-2}+\ldots+\tilde{A}_{p-2} h\right)=h^{p}+A_{1} h^{p-1}+A_{2} h^{p-2}+\ldots+A_{p-1} h
\end{gathered}
$$

If $A_{1}=0$ we continue to calculate to the form $f$ of the rank of $2 p-4$ and we get

$$
\left(\begin{array}{l}
p \\
4
\end{array}\right)(X Y)^{p-4} h_{1}^{2}+A_{2}(X Y)^{p-2}=f_{2 p-4}
$$

Next we proceed the same way as with the constant $A_{1}$. This ends the first part of the proof.

Let $p \geq 2$. We assume that the formula (3) and (4) are true for exponents $q=1, \ldots, p-1$. We will prove that for $q=p$ the formulas are also true. Let's save again the formulas (1) and (2) for $q=p$ 


$$
f=(X Y)^{p}+\left.f_{2 p-1}\right|^{1)}+\left.f_{2 p-2}\right|^{2)}+\left.f_{2 p-3}\right|^{3)}+\ldots+f_{1}
$$

and

$$
h=(X Y)^{p}+\left.h_{2 p-1}\right|^{1)}+\left.h_{2 p-2}\right|^{2)}+\left.h_{2 p-3}\right|^{3)}+\ldots+h_{1}
$$

We have consecutively

$$
\text { 1) } \operatorname{Jac}\left((X Y)^{p}, h_{2 p-1}\right)=\operatorname{Jac}\left((X Y)^{p}, f_{2 p-1}\right)
$$

so

$$
p(X Y)^{p-1} \operatorname{Jac}\left(X Y, h_{2 p-1}\right)=p(X Y)^{p-1} \operatorname{Jac}\left(X Y, f_{2 p-1}\right)
$$

and

$$
\operatorname{Jac}\left(X Y, h_{2 p-1}\right)=\operatorname{Jac}\left(X Y, f_{2 p-1}\right)
$$

finally

$$
h_{2 p-1}=f_{2 p-1}
$$

2) $\operatorname{Jac}\left((X Y)^{p}, h_{2 p-2}\right)+\operatorname{Jac}\left(f_{2 p-1}, h_{2 p-1}\right)=\operatorname{Jac}\left((X Y)^{p}, f_{2 p-2}\right)$

where

$$
\operatorname{Jac}\left(f_{2 p-1}, h_{2 p-1}\right)=\operatorname{Jac}\left(h_{2 p-1}, h_{2 p-1}\right)=0
$$

therefore

$$
p(X Y)^{p-1} \operatorname{Jac}\left(X Y, h_{2 p-2}\right)=p(X Y)^{p-1} \operatorname{Jac}\left(X Y, f_{2 p-2}\right)
$$

and

$$
\operatorname{Jac}\left(X Y, h_{2 p-2}\right)=\operatorname{Jac}\left(X Y, f_{2 p-2}\right)
$$

what gives

$$
h_{2 p-2}+A_{1}(X Y)^{p-1}=f_{2 p-2}
$$

We insert (28), (33) to (24) and we get

$$
f=(X Y)^{p}+h_{2 p-1}+h_{2 p-2}+A_{1}(X Y)^{p-1}+f_{2 p-3}+\ldots+f_{1}
$$

and

$$
h=(X Y)^{p}+h_{2 p-1}+h_{2 p-2}+h_{2 p-3}+\ldots+h_{1}
$$

We subtract

$$
f-h=A_{1}(X Y)^{p-1}+\left(f_{2 p-3}-h_{2 p-3}\right)+\ldots+\left(f_{1}-h_{1}\right)
$$

Let $A_{1} \neq 0$. We assume 


$$
\tilde{f}=\frac{1}{A_{1}}(f-h)=(X Y)^{p-1}+\frac{1}{A_{1}}\left(f_{2 p-3}-h_{2 p-3}\right)+\ldots+\frac{1}{A_{1}}\left(f_{1}-h_{1}\right)=(X Y)^{p-1}+\tilde{f}_{2 p-3}+\ldots . .+\tilde{f}_{1}
$$

Then

$$
\operatorname{Jac}(h, \tilde{f})=-\frac{1}{A_{1}} \operatorname{Jac}(f, h)=\text { const }
$$

Now we apply the induction assumption for exponent $p-1$, converting $f$ to $h$ and $h$ for $\tilde{f}$. Therefore $(X Y)^{p-2} \mid \tilde{f}_{2 p-3}$, which allows us to determine the form $\tilde{f}_{1}$ by the formula $\tilde{f}_{2 p-3}=(X Y)^{p-2} \hat{f}_{1}$. We have at this point

$$
h=\left(X Y+\frac{1}{p-1} \tilde{f}_{1}\right)^{p}+B_{1}\left(X Y+\frac{1}{p-1} \tilde{f}_{1}\right)^{p-1}+\ldots+B_{p-1}\left(X Y+\frac{1}{p-1} \tilde{f}_{1}\right)
$$

and

$$
\tilde{f}=\left(X Y+\frac{1}{p-1} \tilde{f}_{1}\right)^{p-1}+\tilde{A}_{1}\left(X Y+\frac{1}{p-1} \tilde{f}_{1}\right)^{p-1}+\ldots+\tilde{A}_{p-2}\left(X Y+\frac{1}{p-1} \tilde{f}_{1}\right)
$$

for some constants $B_{1}, \ldots, B_{p-1} ; \tilde{A}_{1}, \ldots, \tilde{A}_{p-2}$. Moreover

$$
h_{2 p-1}=\frac{p}{p-1}(X Y)^{p-1} \tilde{f}_{1}
$$

so $(X Y)^{p-1} \mid h_{2 p-1}$. This allows us to determine the form $\hat{h}_{1}$ by the formula $h_{2 p-1}=(X Y)^{p-1} \hat{h}_{1}$. From the formula (42) we get

$$
\frac{1}{p} \hat{h}_{1}=\frac{1}{p-1} \tilde{f}_{1}
$$

Thus

$$
\tilde{f}=\left(X Y+\frac{1}{p} \hat{h}_{1}\right)^{p-1}+\tilde{A}_{1}\left(X Y+\frac{1}{p} \hat{h}_{1}\right)^{p-2}+\ldots+\tilde{A}_{p-2}\left(X Y+\frac{1}{p} \hat{h}_{1}\right)
$$

and

$$
h=\left(X Y+\frac{1}{p} \hat{h}_{1}\right)^{p}+B_{1}\left(X Y+\frac{1}{p} \hat{h}_{1}\right)^{p-1}+\ldots+B_{p-1}\left(X Y+\frac{1}{p} \hat{h}_{1}\right)
$$

Finally

$$
\begin{aligned}
& f=h+A_{1} \tilde{f}= \\
& =h+A_{1}\left[\left(X Y+\frac{1}{p} \hat{h}_{1}\right)^{p-1}+\tilde{A}_{1}\left(X Y+\frac{1}{p} \hat{h}_{1}\right)^{p-2}+\ldots+\tilde{A}_{p-2}\left(X Y+\frac{1}{p} \hat{h}_{1}\right)\right]= \\
& =\left(X Y+\frac{1}{p} \hat{h}_{1}\right)^{p}+A_{1}\left(X Y+\frac{1}{p} \hat{h}_{1}\right)^{p-1}+\left(B_{1}+A_{1} \tilde{A}_{1}\right)\left(X Y+\frac{1}{p} \hat{h}_{1}\right)^{p-2}+\ldots+\left(B_{p-1}+A_{1} \tilde{A}_{p-2}\right)\left(X Y+\frac{1}{p} \hat{h}_{1}\right)= \\
& =\left(X Y+\frac{1}{p} \hat{h}_{1}\right)^{p}+A_{1}\left(X Y+\frac{1}{p} \hat{h}_{1}\right)^{p-1}+A_{2}\left(X Y+\frac{1}{p} \hat{h}_{1}\right)^{p-2}+\ldots+A_{p-1}\left(X Y+\frac{1}{p} \hat{h}_{1}\right)
\end{aligned}
$$

If $A_{1}=0$ we continue to calculate to the form $f$ of the rank of $2 p-4$ and we get 


$$
h_{2 p-4}+A_{2}(X Y)^{p-2}=f_{2 p-4}
$$

Then we proceed in the same way as the constant $A_{1}$ and for the next constants. This completes the second and the last part of the proof.

Theorem II. Let

$$
\begin{aligned}
& f=\left(X^{k} Y^{l}\right)^{p}+f_{(k+l) p-1}+f_{(k+l) p-2}+\ldots+f_{(k+l)(p-1)+1}+\ldots+f_{1} \\
& h=\left(X^{k} Y^{l}\right)^{q}+h_{(k+l) q-1}+h_{(k+l) q-2}+\ldots+h_{(k+l)(q-1)+1}+\ldots+h_{1}
\end{aligned}
$$

where $k>l$ ( $k$ and $l$ are relativity prim) and $p \geq q \geq 1$.

If $\operatorname{Jac}(f, h)=$ const $=\operatorname{Jac}\left(f_{1}, h_{1}\right)$ then exist the forms $\hat{h}_{k+l-1}, \hat{h}_{k+l-2}, \ldots, \hat{h}_{1}$ for which

$$
\begin{aligned}
& f=\left(X^{k} Y^{l}+\frac{1}{q} \hat{h}_{k+l-1}+\frac{1}{q} \hat{h}_{k+l-2}+\ldots+\frac{1}{q} \hat{h}_{1}\right)^{p} \\
& +A_{1}\left(X^{k} Y^{l}+\frac{1}{q} \hat{h}_{k+l-1}+\frac{1}{q} \hat{h}_{k+l-2}+\ldots+\frac{1}{q} \hat{h}_{1}\right)^{p-1} \\
& +A_{p-1}\left(X^{k} Y^{l}+\frac{1}{q} \hat{h}_{k+l-1}+\frac{1}{q} \hat{h}_{k+l-2}+\ldots+\frac{1}{q} \hat{h}_{1}\right)
\end{aligned}
$$

and

$$
\begin{aligned}
& h=\left(X^{k} Y^{l}+\frac{1}{q} \hat{h}_{k+l-1}+\frac{1}{q} \hat{h}_{k+l-2}+\ldots+\frac{1}{q} \hat{h}_{1}\right)^{q} \\
& +B_{1}\left(X^{k} Y^{l}+\frac{1}{q} \hat{h}_{k+l-1}+\frac{1}{q} \hat{h}_{k+l-2}+\ldots+\frac{1}{q} \hat{h}_{1}\right)^{q-1} \\
& +B_{q-1}\left(X^{k} Y^{l}+\frac{1}{q} \hat{h}_{k+l-1}+\frac{1}{q} \hat{h}_{k+l-2}+\ldots+\frac{1}{q} \hat{h}_{1}\right)
\end{aligned}
$$

for some constants $A_{1}, \ldots, A_{p-1}$ and $B_{1}, \ldots, B_{q-1}$

In the proof we will use the following observation.

Observation. Let $\left(g_{k}+g_{k-1}+\ldots+g_{1}\right)^{p}=\hat{g}_{k p}+\hat{g}_{k p-1}+\ldots+\hat{g}_{p}=\sum_{j=0}^{(k-1) p} \hat{g}_{k p-j}$

Then, for $j=0, \ldots,(k-1) p$, we have

$$
\hat{g}_{k p-j}=\sum_{\substack{p_{k-1}+2 p_{k-2}+\ldots+(k-1) p_{1}=j \\ p_{k}+p_{k-1}+\ldots+p_{1}=p, p_{j \geq 0}}} \frac{p !}{p_{k} ! p_{k-1} ! \ldots p_{1} !} g_{k}^{p_{k}} g_{k-1}^{p_{k-1} \ldots g_{1}^{p_{1}}}
$$

Proof of theorem. For $q=1$ the theorem is true. Indeed, let $p \geq 1$. When 


$$
f=\left(X^{k} Y^{l}\right)^{p}+f_{(k+l) p-1}+f_{(k+l) p-2}+\ldots+f_{(k+l)(p-1)+1}+\ldots+f_{1}
$$

and

$$
h=X^{k} Y^{l}+h_{k+l-1}+h_{k+l-2}+\ldots+h_{1}
$$

For $p=1$ the theorem is true, because then $f=h$. In fact, for $p=1$, we have

$$
\begin{aligned}
& f=X^{k} Y^{l}+\left.f_{k+l-1}\right|^{1)}+\left.f_{k+l-2}\right|^{2)}+\ldots+\left.f_{1}\right|^{k+l-1} \\
& h=X^{k} Y^{l}+\left.h_{k+l-1}\right|^{1)}+\left.h_{k+l-2}\right|^{2)}+\ldots+\left.h_{1}\right|^{k+l-1}
\end{aligned}
$$

Because $\operatorname{Jac}(f, h)=$ const then

$$
\text { 1) } \operatorname{Jac}\left(X^{k} Y^{l}, h_{k+l-1}\right)=\operatorname{Jac}\left(X^{k} Y^{l}, f_{k+l-1}\right)
$$

So

$$
h_{k+l-1}=f_{k+l-1}
$$

Next

2) $\operatorname{Jac}\left(X^{k} Y^{l}, h_{k+l-2}\right)+\operatorname{Jac}\left(f_{k+l-1}, h_{k+l-1}\right)=\operatorname{Jac}\left(X^{k} Y^{l}, f_{k+l-2}\right)$

where $\operatorname{Jac}\left(f_{k+l-1}, h_{k+l-1}\right)=0$ according to the formula (59). Consequently

$$
h_{k+l-2}=f_{k+l-2}
$$

By analogy, in step $k+l-1$, we receive

$$
h_{1}=f_{1}
$$

Therefore $f=h$.

Now let $p \geq 2$. We assume that the formulas (50) is true for exponents $1, \ldots$, $p-1$, and we prove that it is true for $p$.

Really, let

$$
f=\left(X^{k} Y^{l}\right)^{p}+\left.f_{(k+l) p-1}\right|^{1)}+\left.f_{(k+l) p-2}\right|^{2)}+\ldots+\left.f_{(k+l)(p-1)+1}\right|^{k+l-2)}+\left.f_{(k+l)(p-1)}\right|^{k+l-1)}+\ldots+\left.f_{1}\right|^{(k+l) p-1)}
$$

and

$$
h=X^{k} Y^{l}+\left.h_{k+l-1}\right|^{1)}+\left.h_{k+l-2}\right|^{2)}+\ldots \quad+\left.h_{2}\right|^{k+l-2)}+\left.\quad h_{1}\right|^{k+l-1)}+\ldots+0
$$

We have

$$
\text { 1) } \operatorname{Jac}\left(\left(X^{k} Y^{l}\right)^{p}, h_{k+l-1}\right)=\operatorname{Jac}\left(X^{k} Y^{l}, f_{(k+l) p-1}\right)
$$

so 


$$
p\left(X^{k} Y^{l}\right)^{p-1} \operatorname{Jac}\left(X^{k} Y^{l}, h_{k+l-1}\right)=\operatorname{Jac}\left(X^{k} Y^{l}, f_{(k+l) p-1}\right)
$$

therefore

$$
p\left(X^{k} Y^{l}\right)^{p-1} h_{k+l-1}=f_{(k+l) p-1}
$$

Next

$$
\text { 2) } \operatorname{Jac}\left(\left(X^{k} Y^{l}\right)^{p}, h_{k+l-2}\right)+\underbrace{\operatorname{Jac}\left(f_{(k+l) p-1}, h_{k+l-1}\right)}_{1^{\circ}}=\operatorname{Jac}\left(X^{k} Y^{l}, f_{(k+l) p-2}\right)
$$

where

$$
\begin{aligned}
1^{\circ} & =\operatorname{Jac}\left(f_{(k+l) p-1}, h_{k+l-1}\right)=\operatorname{Jac}\left(p\left(X^{k} Y^{l}\right)^{p-1} h_{k+l-1}, h_{k+l-1}\right)= \\
& =p h_{k+l-1} \operatorname{Jac}\left(\left(X^{k} Y^{l}\right)^{p-1}, h_{k+l-1}\right)=p(p-1)\left(X^{k} Y^{l}\right)^{p-2} h_{k+l-1} \operatorname{Jac}\left(X^{k} Y^{l}, h_{k+l-1}\right)
\end{aligned}
$$

and back to the previous formula, we have

$$
p\left(X^{k} Y^{l}\right)^{p-1} \operatorname{Jac}\left(X^{k} Y^{l}, h_{k+l-2}\right)+p(p-1)\left(X^{k} Y^{l}\right)^{p-2} h_{k+l-1} \operatorname{Jac}\left(X^{k} Y^{l}, h_{k+l-1}\right)=\operatorname{Jac}\left(X^{k} Y^{l}, f_{(k+l) p-2}\right)
$$

so

$$
p\left(X^{k} Y^{l}\right)^{p-1} h_{k+l-2}+\frac{p(p-1)}{2}\left(X^{k} Y^{l}\right)^{p-2} h_{k+l-1}^{2}=f_{(k+l) p-2}
$$

Continuing calculations up to form $f_{(k+l)(p-1)}$ we obtain

$$
f_{(k+l)(p-1)}=\hat{h}_{(k+l)(p-1)}+A_{1}\left(X^{k} Y^{l}\right)^{p-1}
$$

where e. g.

I. for $p \geq k+l$ we have

1. if $k+l$ is odd

$$
\begin{aligned}
& \hat{h}_{(k+l)(p-1)}=\sum_{\substack{p_{k+l-1}+2 p_{k+l-2}+\ldots+(k+l-1) p_{1}=k+l \\
p_{k+l}+p_{k+l-1}+\ldots+p_{1}=p, p_{j} \geq 0}} \frac{p !}{p_{k+l} ! p_{k+l-1} ! \ldots p_{1} !}\left(X^{k} Y^{l}\right)^{p_{k+l}} h_{k+l-1}^{p_{k+l-1} \ldots h_{1}^{p_{1}}}= \\
& =\frac{p !}{(p-(k+l)) !(k+l) !}\left(X^{k} Y^{l}\right)^{p-(k+l)} h_{k+l-1}^{k+l}+\ldots+\frac{p !}{(p-2) !}\left(X^{k} Y^{l}\right)^{p-2} h_{\frac{k+l-1}{2}+1} h_{\frac{k+l-1}{2}}
\end{aligned}
$$

2. if $k+l$ is even

$$
\begin{aligned}
& \hat{h}_{(k+l)(p-1)}=\sum_{\substack{p_{k+l-1}+2 p_{k+l-2}+\ldots+(k+l-1) p_{1}=k+l \\
p_{k+l}+p_{k+l-1}+\ldots+p_{1}=p, p_{j} \geq 0}} \frac{p !}{p_{k+l} ! p_{k+l-1} ! \ldots p_{1} !}\left(X^{k} Y^{l}\right)^{p_{k+l}} h_{k+l-1}^{p_{k+l-1} \ldots} h_{1}^{p_{1}}= \\
& =\frac{p !}{(p-(k+l)) !(k+l) !}\left(X^{k} Y^{l}\right)^{p-(k+l)} h_{k+l-1}^{k+l}+\ldots+\frac{p !}{2 !(p-2) !}\left(X^{k} Y^{l}\right)^{p-2} h_{\frac{k+l}{2}}^{2}
\end{aligned}
$$

II. for $p<k+l$ we have 


$$
\hat{h}_{(k+l)(p-1)}=\sum_{p_{k+l-1}+2 p_{k+l-2}+\ldots+(k+l-1) p_{1}=k+l} \frac{p !}{p_{k+l}+2 p_{k+l-1}+\ldots+p_{1} \leq k+l-1, p_{j} \geq 0} \frac{p}{p_{k+l} ! p_{k+l-1} ! \ldots p_{1} !}\left(X^{k} Y^{l}\right)^{p_{k+l}} h_{k+l-1}^{p_{k+l-1} \ldots} h_{1}^{p_{1}}
$$

according to the above observation.

On the other hand we get

$$
\begin{aligned}
& h^{p}=\left(X^{k} Y^{l}+h_{k+l-1}+h_{k+l-2}+\ldots+h_{k}+\ldots+h_{l}+\ldots+h_{1}\right)^{p}= \\
& =\left(X^{k} Y^{l}\right)^{p}+p\left(X^{k} Y^{l}\right)^{p-1} h_{k+l-1}+\left[p\left(X^{k} Y^{l}\right)^{p-1} h_{k+l-2}+\frac{p(p-1)}{2}\left(X^{k} Y^{l}\right)^{p-2} h_{k+l-1}^{2}\right]+\ldots+\hat{h}_{(k+l)(p-1)}
\end{aligned}
$$

Thus, the formulas (67), (71), (72) allow to write

$$
\begin{aligned}
& f=\left(X^{k} Y^{l}\right)^{p}+p\left(X^{k} Y^{l}\right)^{p-1} h_{k+l-1}+p\left(X^{k} Y^{l}\right)^{p-1} h_{k+l-2}+ \\
& +\frac{p(p-1)}{2}\left(X^{k} Y^{l}\right)^{p-2} h_{k+l-1}^{2}+\ldots+\hat{h}_{(k+l)(p-1)}+A_{1}\left(X^{k} Y^{l}\right)^{p-1}+f_{(k+l)(p-1)-1}+\ldots+f_{p}+f_{p-1}+\ldots+f_{1}
\end{aligned}
$$

Hence

$$
f-h^{p}=A_{1}\left(X^{k} Y^{l}\right)^{p-1}+\left(f_{(k+l)(p-1)-1}-\hat{h}_{(k+l)(p-1)-1}\right)+\ldots+\left(f_{p}-h_{1}^{p}\right)+f_{p-1}+\ldots+f_{1}
$$

Let $A_{1} \neq 0$. Denote

$$
\begin{aligned}
& \tilde{f}=\frac{1}{A_{1}}\left(f-h^{p}\right)= \\
& =\left(X^{k} Y^{l}\right)^{p-1}+\frac{1}{A_{1}}\left(f_{(k+l)(p-1)-1}-\hat{h}_{(k+l)(p-1)-1}\right)+\ldots+\frac{1}{A_{1}}\left(f_{p}-h_{1}^{p}\right)+\frac{1}{A_{1}} f_{p-1}+\ldots+\frac{1}{A_{1}} f_{1}= \\
& =\left(X^{k} Y^{l}\right)^{p-1}+\tilde{f}_{(k+l)(p-1)-1}+\ldots+\tilde{f}_{p}+\tilde{f}_{p-1}+\ldots+\tilde{f}_{1}
\end{aligned}
$$

Using the induction assumption for exponent $p-1$ we have

$$
\tilde{f}=h^{p-1}+\tilde{A}_{1} h^{p-2}+\ldots+\tilde{A}_{p-2} h
$$

for some constants $\tilde{A}_{1}, \ldots, \tilde{A}_{p-2}$. Therefore

$$
\begin{aligned}
f & =h^{p}+A_{1} \tilde{f}=h^{p}+A_{1}\left(h^{p-1}+\tilde{A}_{1} h^{p-2}+\ldots+\tilde{A}_{p-2} h\right)= \\
& =h^{p}+A_{1} h^{p-1}+A_{1} \tilde{A}_{1} h^{p-2}+\ldots+A_{1} \tilde{A}_{p-2} h= \\
& =h^{p}+A_{1} h^{p-1}+A_{2} h^{p-2}+\ldots+A_{p-1} h
\end{aligned}
$$

If $A_{1}=0$ we continue to calculate to the form $f$ of the rank of $(k+l)(p-2)$ and we get

$$
\hat{h}_{(k+l)(p-2)}+A_{2}\left(X^{k} Y^{l}\right)^{p-2}=f_{(k+l)(p-2)}
$$

With the constant $A_{2}$ we proceed the same way as with the constant $A_{1}$. What ends the first part of the proof. 
Let $p \geq 2$. We assume that the formula (50) and (51) are true for exponents $1 \leq q \leq p-1$. We will prove that for $q=p$ the formulas are also true. Then we have

$$
f=\left(X^{k} Y^{l}\right)^{p}+\left.f_{p(k+l)-1}\right|^{1)}+\ldots+\left.f_{(p-1)(k+l)+1}\right|^{k+l-1)}+\left.f_{(p-1)(k+l)}\right|^{k+l)}+\left.f_{(p-1)(k+l)-1}\right|^{k+l+1)}+\ldots+f_{1}
$$

and

$$
h=\left(X^{k} Y^{l}\right)^{p}+\left.h_{p(k+l)-1}\right|^{1)}+\ldots+\left.h_{(p-1)(k+l)+1}\right|^{k+l-1)}+\left.h_{(p-1)(k+l)}\right|^{k+l)}+\left.h_{(p-1)(k+l)-1}\right|^{k+l+1)}+\ldots+h_{1}
$$

Because $\operatorname{Jac}(f, h)=$ const , we have consecutively

$$
\text { 1) } \operatorname{Jac}\left(\left(X^{k} Y^{l}\right)^{p}, h_{p(k+l)-1}\right)=\operatorname{Jac}\left(\left(X^{k} Y^{l}\right)^{p}, f_{p(k+l)-1}\right)
$$

so

$$
p\left(X^{k} Y^{l}\right)^{p-1} \operatorname{Jac}\left(X^{k} Y^{l}, h_{p(k+l)-1}\right)=p\left(X^{k} Y^{l}\right)^{p-1} \operatorname{Jac}\left(X^{k} Y^{l}, f_{p(k+l)-1}\right)
$$

and

$$
\operatorname{Jac}\left(X^{k} Y^{l}, h_{p(k+l)-1}\right)=\operatorname{Jac}\left(X^{k} Y^{l}, f_{p(k+l)-1}\right)
$$

finally

$$
f_{p(k+l)-1}=h_{p(k+l)-1}
$$

We proceed similarly to step $k+l-1$ and we also receive

$$
f_{(p-1)(k+l)+1}=h_{(p-1)(k+l)+1}
$$

In the step $k+l$ we have

$$
f_{(p-1)(k+l)}=h_{(p-1)(k+l)}+A_{1}\left(X^{k} Y^{l}\right)^{p-1}
$$

Now we subtract

$$
f-h=A_{1}\left(X^{k} Y^{l}\right)^{p-1}+\left(f_{(p-1)(k+l)-1}-h_{(p-1)(k+l)-1}\right)+\ldots+\left(f_{1}-h_{1}\right)
$$

Let $A_{1} \neq 0$. We define

$$
\begin{aligned}
\tilde{f} & =\frac{1}{A_{1}}(f-h)=\left(X^{k} Y^{l}\right)^{p-1}+\frac{1}{A_{1}}\left(f_{(p-1)(k+l)-1}-h_{(p-1)(k+l)-1}\right)+\ldots+\frac{1}{A_{1}}\left(f_{1}-h_{1}\right)= \\
& =\left(X^{k} Y^{l}\right)^{p-1}+\tilde{f}_{(p-1)(k+l)-1}+\ldots .+\tilde{f}_{1}
\end{aligned}
$$

Then

$$
\operatorname{Jac}(h, \tilde{f})=\frac{1}{A_{1}} \operatorname{Jac}(h, f-h)=\frac{1}{A_{1}} \operatorname{Jac}(h, f)=\text { const }
$$

wherein

$$
h=\left(X^{k} Y^{l}\right)^{p}+h_{(k+l) p-1}+h_{(k+l) p-2}+\ldots+h_{(k+l)(p-1)+1}+h_{(k+l)(p-1)}+\ldots+h_{1}
$$


and

$$
\tilde{f}=\left(X^{k} Y^{l}\right)^{p-1}+\tilde{f}_{(p-1)(k+l)-1}+\tilde{f}_{(p-1)(k+l)-2}+\ldots .+\tilde{f}_{1}
$$

We receive the assumption for exponent $q=p-1$. When exist the forms $\hat{f}_{k+l-1}, \hat{f}_{k+l-1}, \ldots, \hat{f}_{1}$ for which

$$
h=\left(X^{k} Y^{l}+\frac{1}{p-1} \hat{f}_{k+l-1}+\ldots+\frac{1}{p-1} \hat{f}_{1}\right)^{p}+B_{1}\left(X^{k} Y^{l}+\frac{1}{p-1} \hat{f}_{k+l-1}+\ldots+\frac{1}{p-1} \hat{f}_{1}\right)^{p-1}+\ldots
$$

and

$$
\tilde{f}=\left(X^{k} Y^{l}+\frac{1}{p-1} \hat{f}_{k+l-1}+\ldots+\frac{1}{p-1} \hat{f}_{1}\right)^{p-1}+\tilde{A}_{1}\left(X^{k} Y^{l}+\frac{1}{p-1} \hat{f}_{k+l-1}+\ldots+\frac{1}{p-1} \hat{f}_{1}\right)^{p-2}+\ldots
$$

The first term in (96) after development give the formulas for successive homogeneous components of the polynomial $h$ in (94). So

$$
\frac{p}{p-1}\left(X^{k} Y^{l}\right)^{p-1} \hat{f}_{k+l-1}=h_{(k+l) p-1}
$$

Therefore $\left(X^{k} Y^{l}\right)^{p-1} \mid h_{(k+l) p-1}$ and we have the formula for the form $\hat{h}_{k+l-1}$

$$
h_{(k+l) p-1}=\left(X^{k} Y^{l}\right)^{p-1} \hat{h}_{k+l-1}
$$

Furthermore

$$
\frac{1}{p-1} \hat{f}_{k+l-1}=\frac{1}{p} \hat{h}_{k+l-1}
$$

Therefore now

$$
h=\left(X^{k} Y^{l}+\frac{1}{p} \hat{h}_{k+l-1}+\frac{1}{p-1} \hat{f}_{k+l-2}+\ldots+\frac{1}{p-1} \hat{f}_{1}\right)^{p}+B_{1}\left(X^{k} Y^{l}+\frac{1}{p} \hat{h}_{k+l-1}+\frac{1}{p-1} \hat{f}_{k+l-2}+\ldots+\frac{1}{p-1} \hat{f}_{1}\right)^{p-1}+\ldots
$$

and

$$
\tilde{f}=\left(X^{k} Y^{l}+\frac{1}{p} \hat{h}_{k+l-1}+\frac{1}{p-1} \hat{f}_{k+l-2}+\ldots+\frac{1}{p-1} \hat{f}_{1}\right)^{p-1}+\tilde{A}_{1}\left(X^{k} Y^{l}+\frac{1}{p} \hat{h}_{k+l-1}+\frac{1}{p-1} \hat{f}_{k+l-2}+\ldots+\frac{1}{p-1} \hat{f}_{1}\right)^{p-2}+\ldots
$$

Next homogeneous component of the polynomial $h$ in (94) is equal now

$$
\frac{p}{p-1}\left(X^{k} Y^{l}\right)^{p-1} \hat{f}_{k+l-2}+\frac{p-1}{2 p}\left(X^{k} Y^{l}\right)^{p-2} \hat{h}_{k+l-1}^{2}=h_{(k+l) p-2}
$$

Therefore

$$
\frac{p}{p-1}\left(X^{k} Y^{l}\right)^{p-1} \hat{f}_{k+l-2}=h_{(k+l) p-2}-\frac{p-1}{2 p}\left(X^{k} Y^{l}\right)^{p-2} \hat{h}_{k+l-1}^{2}=\tilde{h}_{(k+l) p-2}
$$

and $\left(X^{k} Y^{l}\right)^{p-1} \mid \tilde{h}_{(k+l) p-1}$ so the formula for the form $\hat{h}_{k+l-2}$ is ready 


$$
\tilde{h}_{(k+l) p-2}=\left(X^{k} Y^{l}\right)^{p-1} \hat{h}_{k+l-2}
$$

We get the same

$$
\frac{1}{p-1} \hat{f}_{k+l-2}=\frac{1}{p} \hat{h}_{k+l-2}
$$

After $k+l-2$ steps we receive

$$
\begin{aligned}
h & =\left(X^{k} Y^{l}+\frac{1}{p} \hat{h}_{k+l-1}+\frac{1}{p} \hat{h}_{k+l-2}+\ldots+\frac{1}{p} \hat{h}_{2}+\frac{1}{p-1} \hat{f}_{1}\right)^{p} \\
& +B_{1}\left(X^{k} Y^{l}+\frac{1}{p} \hat{h}_{k+l-1}+\frac{1}{p} \hat{h}_{k+l-2}+\ldots+\frac{1}{p} \hat{h}_{2}+\frac{1}{p-1} \hat{f}_{1}\right)^{p-1}+\ldots
\end{aligned}
$$

and

$$
\begin{aligned}
\tilde{f} & =\left(X^{k} Y^{l}+\frac{1}{p} \hat{h}_{k+l-1}+\frac{1}{p} \hat{h}_{k+l-2}+\ldots+\frac{1}{p} \hat{h}_{2}+\frac{1}{p-1} \hat{f}_{1}\right)^{p-1} \\
& +\tilde{A}_{1}\left(X^{k} Y^{l}+\frac{1}{p} \hat{h}_{k+l-1}+\frac{1}{p} \hat{h}_{k+l-2}+\ldots+\frac{1}{p} \hat{h}_{2}+\frac{1}{p-1} \hat{f}_{1}\right)^{p-2}+\ldots
\end{aligned}
$$

The homogeneous component of the polynomial $h$ of degree $(k+l)(p-1)+1$ in (94) is equal now

$$
\begin{aligned}
& \quad \sum_{\substack{p_{k+l-1}+2 p_{k+l-2}+\ldots+(k+l-1) p_{1}=k+l-1 \\
p_{k+l}+p_{k+l-1}+\ldots+p_{1}=p, p_{j} \geq 0}} \frac{p !}{p_{k+l} ! p_{k+l-1} ! \ldots p_{1} !}\left(X^{k} Y^{l}\right)^{p_{k+l}}\left(\frac{1}{p} \hat{h}_{k+l-1}\right)^{p_{k+l-1}} \cdots\left(\frac{1}{p} \hat{h}_{2}\right)^{p_{2}}\left(\frac{1}{p-1} \hat{f}_{1}\right)^{p_{1}}= \\
& =\frac{p}{p-1}\left(X^{k} Y^{l}\right)^{p-1} \hat{f}_{1}+\sum_{\substack{p_{k+l-1}+2 p_{k+l-2}+\ldots+(k+l-2) p_{2}=k+l-1 \\
p_{k+l}+p_{k+l-1}+\ldots+p_{2}=p, p_{j} \geq 0}} \frac{p !}{p_{k+l} ! p_{k+l-1} ! \ldots p_{2} !}\left(X^{k} Y^{l}\right)^{p_{k+l}}\left(\frac{1}{p} \hat{h}_{k+l-1}\right)^{p_{k+l-1}} \ldots\left(\frac{1}{p} \hat{h}_{2}\right)^{p_{2}} \\
& =h_{(k+l)(p-1)+1}
\end{aligned}
$$

So

$$
\begin{aligned}
& \frac{p}{p-1}\left(X^{k} Y^{l}\right)^{p-1} \hat{f}_{1}= \\
& =h_{(k+l)(p-1)+1}-\sum_{\substack{p_{k+l-1}+2 p_{k+l-2}+\ldots+(k+l-2) p_{2}=k+l-1 \\
p_{k+l}+p_{k+l-1}+\ldots+p_{2}=p, p_{j} \geq 0}} \frac{p !}{p_{k+l} ! p_{k+l-1} ! \ldots p_{2} !}\left(X^{k} Y^{l}\right)^{p_{k+l}}\left(\frac{1}{p} \hat{h}_{k+l-1}\right)^{p_{k+l-1}} \ldots\left(\frac{1}{p} \hat{h}_{2}\right)^{p_{2}} \\
& =\tilde{h}_{(k+l)(p-1)+1}
\end{aligned}
$$

and the formula for the form $\hat{h}_{1}$ is ready

$$
\tilde{h}_{(k+l)(p-1)+1}=\left(X^{k} Y^{l}\right)^{p-1} \hat{h}_{1}
$$

Therefore

We get finally

$$
\frac{1}{p-1} \hat{f}_{1}=\frac{1}{p} \hat{h}_{1}
$$


$h=\left(X^{k} Y^{l}+\frac{1}{p} \hat{h}_{k+l-1}+\frac{1}{p} \hat{h}_{k+l-2}+\ldots+\frac{1}{p} \hat{h}_{2}+\frac{1}{p} \hat{h}_{1}\right)^{p}+B_{1}\left(X^{k} Y^{l}+\frac{1}{p} \hat{h}_{k+l-1}+\frac{1}{p} \hat{h}_{k+l-2}+\ldots+\frac{1}{p} \hat{h}_{2}+\frac{1}{p} \hat{h}_{1}\right)^{p-1}+\ldots$

and

$\tilde{f}=\left(X^{k} Y^{l}+\frac{1}{p} \hat{h}_{k+l-1}+\frac{1}{p} \hat{h}_{k+l-2}+\ldots+\frac{1}{p} \hat{h}_{2}+\frac{1}{p} \hat{h}_{1}\right)^{p-1}+\tilde{A}_{1}\left(X^{k} Y^{l}+\frac{1}{p} \hat{h}_{k+l-1}+\frac{1}{p} \hat{h}_{k+l-2}+\ldots+\frac{1}{p} \hat{h}_{2}+\frac{1}{p} \hat{h}_{1}\right)^{p-2}+\ldots$

So

$$
\begin{aligned}
f & =h+A_{1} \tilde{f}=h+A_{1}\left(X^{k} Y^{l}+\frac{1}{p} \hat{h}_{k+l-1}+\frac{1}{p} \hat{h}_{k+l-2}+\ldots+\frac{1}{p} \hat{h}_{2}+\frac{1}{p} \hat{h}_{1}\right)^{p-1}+ \\
& +A_{1} \tilde{A}_{1}\left(X^{k} Y^{l}+\frac{1}{p} \hat{h}_{k+l-1}+\frac{1}{p} \hat{h}_{k+l-2}+\ldots+\frac{1}{p} \hat{h}_{2}+\frac{1}{p} \hat{h}_{1}\right)^{p-2}+\ldots \\
& =\left(X^{k} Y^{l}+\frac{1}{p} \hat{h}_{k+l-1}+\frac{1}{p} \hat{h}_{k+l-2}+\ldots+\frac{1}{p} \hat{h}_{2}+\frac{1}{p} \hat{h}_{1}\right)^{p}+ \\
& \left(B_{1}+A_{1}\right)\left(X^{k} Y^{l}+\frac{1}{p} \hat{h}_{k+l-1}+\frac{1}{p} \hat{h}_{k+l-2}+\ldots+\frac{1}{p} \hat{h}_{2}+\frac{1}{p} \hat{h}_{1}\right)^{p-1}+ \\
& +\left(B_{2}+A_{1} \tilde{A}_{1}\right)\left(X^{k} Y^{l}+\frac{1}{p} \hat{h}_{k+l-1}+\frac{1}{p} \hat{h}_{k+l-2}+\ldots+\frac{1}{p} \hat{h}_{2}+\frac{1}{p} \hat{h}_{1}\right)^{p-2}+\ldots \\
& =\left(X^{k} Y^{l}+\frac{1}{p} \hat{h}_{k+l-1}+\frac{1}{p} \hat{h}_{k+l-2}+\ldots+\frac{1}{p} \hat{h}_{2}+\frac{1}{p} \hat{h}_{1}\right)^{p}+ \\
& +A_{1}\left(X^{k} Y^{l}+\frac{1}{p} \hat{h}_{k+l-1}+\frac{1}{p} \hat{h}_{k+l-2}+\ldots+\frac{1}{p} \hat{h}_{2}+\frac{1}{p} \hat{h}_{1}\right)^{p-1}+ \\
+ & A_{2}\left(X^{k} Y^{l}+\frac{1}{p} \hat{h}_{k+l-1}+\frac{1}{p} \hat{h}_{k+l-2}+\ldots+\frac{1}{p} \hat{h}_{2}+\frac{1}{p} \hat{h}_{1}\right)^{p-2}+\ldots
\end{aligned}
$$

The formulas (113) and (115) give the result where $A_{1} \neq 0$.

If $A_{1}=0$ we continue to calculate to the form $f$ of the rank of $(k+l)(p-2)$ and we get

$$
h_{(k+l)(p-2)}+A_{2}\left(X^{k} Y^{l}\right)^{p-2}=f_{(k+l)(p-2)}
$$

With the constant $A_{2}$ we proceed the same way as with the constant $A_{1}$. What ends the final part of the proof.

Corollary 1. Under the assumptions of both theorems the polynomials $f$ and $h$ are algebraically depending.

Corollary 2. Not exist invertible polynomial mappings which have two zeros at infinity.

\section{References}

[1] Keller O.H., Ganze Cremona-Transformationen, Math.Phys. 47, 229-306, 1939.

[2] Moh T.T., On analitic irreducibility at infinity of a pencil of curves, J. Proc. Amer. Math. Soc. 44, 22-24, 1974.

[3] Biernat G., The jacobians of lower degrees, Scientific Research of the Institute of Mathematics and Computer Science, 2(1), 19-24, 2003.

[4] Abhyankar S.S., Expansion techniques in algebraic geometry, Tata Inst. Fundamental Research,Bombay, 1977. 
[5] Charzyński Z., Chądzyński J., Skibinski P., A contribution to Keller’s Jacobian Conjecture, Lecture Notes In Math. 1165, Springer-Verlag, Berlin Heidelberg N. York, 36-51, 1985.

[6] Wright D., On the Jacobian conjecture, no. 3, 423-440, Illinois J. Math. 25, 1981.

[7] Van den Essen A., Polynomial automorphisms and the Jacobian conjecture, Progress in Matematics 190, Basel: Birkhäuser Verlag, 2000.

[8] Bass H., Connell E.H., Wright D., The Jacobian conjecture: reduction of degree and formal expansion of the inverse, American Mathematical Society. Bulletin. New Series 7 (2): 287-330, 1982. 